CASE REPORT

\author{
L.-D. Jou \\ A. Mohamed \\ D.H. Lee \\ M.E. Mawad
}

\section{D Rotational Digital Subtraction Angiography May Underestimate Intracranial Aneurysms: Findings from Two Basilar Aneurysms}

\begin{abstract}
SUMmARY: 3D digital subtraction angiography (DSA) allows clinicians to review intracranial aneurysms and other vascular lesions. We report 2 basilar aneurysms that were imaged by both 3D DSA and DynaCT. These 2 techniques produced very different aneurysm appearances. Anterior portions of the aneurysms were invisible on 3D DSA but were revealed by DynaCT. These aneurysms appeared to have been flattened by image artifacts in 3D DSA. Pulsation and gravity are 2 possible causes of aneurysm underestimation.
\end{abstract}

D igital subtraction angiography (DSA) has been the "gold standard" for many vascular and cerebrovascular imaging studies. 3D rotational DSA enables clinicians to review intracranial aneurysms and other vascular lesions thoroughly in an angiography study or during an interventional procedure. 3D rotational DSA was shown to perform better than either MR or CT angiography in 1 experimental study ${ }^{1}$ in which time-offlight MR angiography underestimated aneurysm volumes, whereas CT angiography overestimated. Nevertheless, 3D DSA still is affected by image artifacts that may potentially influence diagnostic accuracy and impact clinical decisions. ${ }^{2}$ The requirement of uniformly distributed contrast agent across the region of interest often cannot be met in some vascular regions, such as at a lateral saccular aneurysm with a narrow neck. ${ }^{3}$ Aneurysms with certain orientation also may not be accessible by contrast injection. This is especially true during endovascular procedures when coils are already inside the aneurysm. Moreover, insufficient contrast influx can introduce image artifacts in 3D DSA. ${ }^{3}$ For example, parent vessels near an aneurysm may appear stenotic. ${ }^{4}$ In this report, we describe 2 cases of basilar aneurysms for which there are considerable differences in aneurysm volume and morphology between 3D DSA and DynaCT (Siemens Medical Solutions, Erlangen, Germany). ${ }^{5}$

\section{Case Report}

The first case was a 79-year-old woman who had a history of hypertension, diabetes, and hyperlipidemia. She was diagnosed with a basilar tip aneurysm after an episode of fainting. The aneurysm was treated with coil embolization without any incidents. Figure 1 shows the lateral and oblique view of the basilar tip aneurysm from 3D DSA and DynaCT. Because both images have the same resolution, a thick section in the middle of the aneurysm was selected for comparison and is presented in Fig 1.

The second case was a 48-year-old man with asthma in whom a basilar trunk aneurysm was diagnosed. His bilobed basilar aneurysm was measured at $9 \times 10 \mathrm{~mm}$ on the right and $10 \times 10 \mathrm{~mm}$ on the left. The patient underwent stent placement and coiling for the basilar

Received January 16, 2007; accepted after revision March 23.

From the Department of Radiology (L.-D.J., D.H.L., M.E.M.), Baylor College of Medicine, Houston, Tex; Siemens Medical Solutions (A.M.), Princeton, NJ; and the Asan Medical Center (D.H.L.), Seoul, South Korea.

Please address correspondence to Liang-Der Jou, PhD, Frensley Center for Imaging Research, Department of Radiology, BCM-360, Baylor College of Medicine, One Baylor Plaza, Houston, TX 77030; e-mail: jou@bcm.tmc.edu

DOI 10.3174/ajnr.A0652 aneurysm. Both 3D DSA and DynaCT images of the aneurysm are shown in Figure 2. The anterior portion of his right-lobed aneurysm was not visible on 3D DSA.

Both patients were imaged with 3D DSA and DynaCT protocols on an AXIOM Artis dBA biplane flat detector system with DynaCT capability (Siemens Medical Solutions). For 3D DSA, $30 \mathrm{~mL}$ of contrast agent (Omnipaque 300; GE Healthcare, Princeton, NJ) was injected at $2.5 \mathrm{~mL} / \mathrm{s}$ for 12 seconds, and 275 projections were acquired in $220^{\circ}$ within 10 seconds to reconstruct $3 \mathrm{D}$ images. There was a 2 -second delay for contrast filling. In DynaCT, $22 \mathrm{~mL}$ of same contrast agent diluted by the saline (25\%) was injected at $1 \mathrm{~mL} / \mathrm{s}$, and 543 projections within $220^{\circ}$ were acquired for reconstruction. Both procedures had a 2 -second $\mathrm{x}$-ray delay and produced 3D images with a $0.4-\mathrm{mm}$ isotropic resolution after reconstruction. The difference in the appearance of aneurysms in DynaCT and 3D DSA after coregistration is presented in Fig 1 for the first case and in Fig 2 for the second. Both cases showed anterior portions of aneurysms in DynaCT, but they were missing from the 3D DSA. In the 3D DSA images, anterior portions of the aneurysms seemed flat on the $\mathrm{X}$-Leonardo workstation (Siemens Medical Solutions); this appearance may be caused by image artifacts.

\section{Discussion}

Presence of artifacts may affect the calculation of aneurysm volume and the determination of packing ratio during an endovascular procedure. In turn, this may influence the clinician's perception about the completion of a procedure. ${ }^{6}$ Aneurysm volumes for these 2 cases were underestimated by approximately $20 \%$ in 3D DSA. The basilar trunk aneurysm in case 2 had a narrow neck, and its image appearance may have been affected by contrast deficit during imaging. The basilar tip aneurysm in case 1 had a wider neck and was directly in the flow path for the basilar artery. Therefore, this artifact cannot be explained simply as insufficient flow into the aneurysm. ${ }^{3}$ The possible mechanisms that resulted in this artifact are the blood flow pulsation and difference in density between blood and contrast medium.

Although contrast medium is injected at a constant rate, blood flow into the aneurysm is pulsatile, resulting in a fluctuation of contrast medium concentration. This can be observed in projective views of unmasked images. Contrast injection also can increase the flow rate considerably in cerebral vessels. The mean flow rate at the basilar artery is approximately $3 \mathrm{~mL} / \mathrm{s}^{7}$; thus, an injection of $2.5 \mathrm{~mL} / \mathrm{s}$ in these cases may have nearly doubled the mean flow rate at the basilar artery. Injection from only one of the vertebral arteries does 

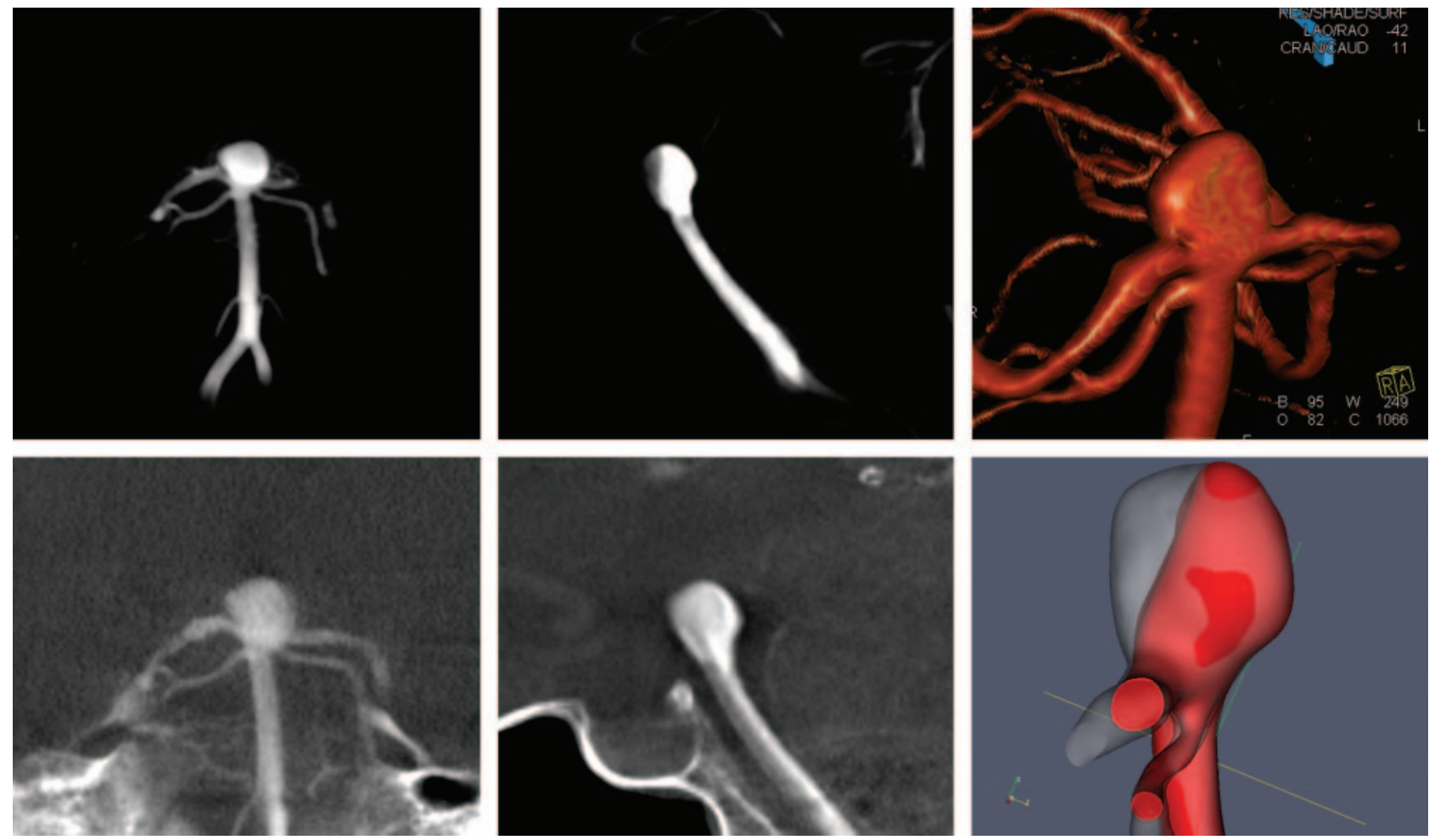

Figure 1. Top row shows the front (left) and lateral views (middle) of 3D DSA and a rendering image (right). Bottom row shows the front (left) and lateral views (middle) of DynaCT images and overlap of basilar tip aneurysm models from DSA and DynaCT images (right). Aneurysm from 3D DSA is in red and aneurysm from DynaCT is in light gray. A large portion of aneurysm from DynaCT is missing in 3D DSA. 3D DSA indicates 3D digital subtraction angiography.
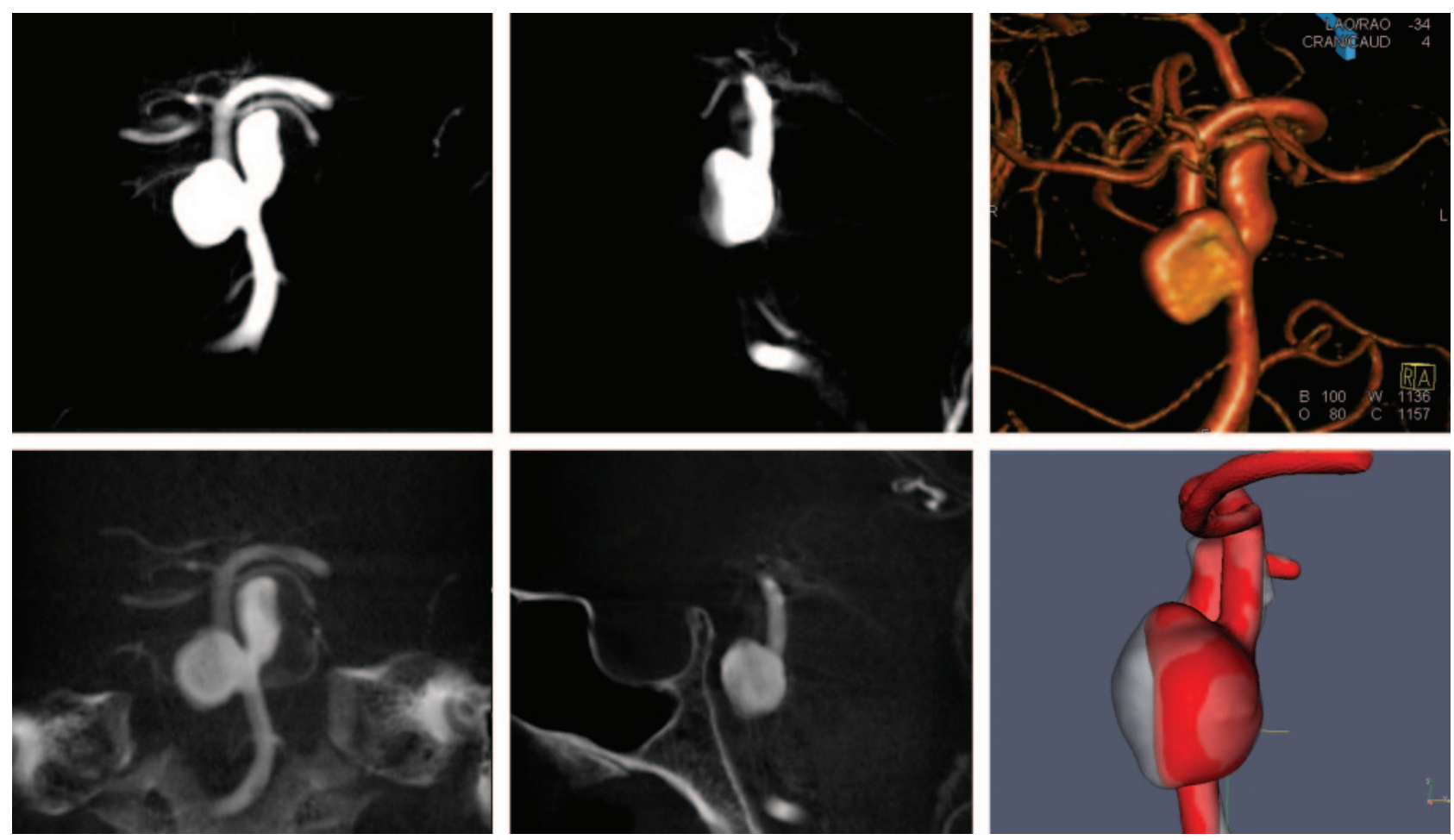

Figure 2. Top row shows the front (left) and lateral views (middle) of 3D DSA and a rendering image (right). Bottom row shows the front (left) and lateral views (middle) of DynaCT images and overlap of basilar trunk aneurysm models from DSA and DynaCT images (right). Aneurysm from 3D DSA is in red, and aneurysm from DynaCT is in light gray. A portion of aneurysm in DynaCT is missing in 3D DSA. 3D DSA indicates 3D digital subtraction angiography.

not produce blood with uniform contrast at the basilar artery, and a portion of the aneurysm consisting of blood mainly from the noninjecting vertebral artery may appear unopacified. 
Gravity also may have played an important role in generating this artifact. The contrast medium used for cerebral angiography has a higher density than the blood and often settles at the posterior part of the aneurysm after washout. ${ }^{8}$ The artifacts in both cases appear at the anterior side of the aneurysms, indicating that it may be difficult for the contrast medium to reach these regions. Given that the specific density of Omnipaque is 1.35 , the specific density of blood injected with contrast medium is 1.18 in 3D DSA and 1.02 in DynaCT for the mean flow rate of $2.5 \mathrm{~mL} / \mathrm{s}$ at the basilar artery. Therefore, the diluted contrast agent used in DynaCT has a density that is closer to that of the blood and may be able to fill the entire aneurysm better than the concentrated contrast medium used in 3D DSA. This may partly explain why the disappearance is always at the anterior side.

In both of these cases, the significant difference between 3D DSA and DynaCT was at the basilar artery. Although the basilar artery is the only artery we have observed so far that is susceptible to this artifact, there is no reason to believe that it is limited to this part of the vasculature. A careful examination of images from 3D DSA and DynaCT at other regions may allow a better understanding of this artifact.

\section{References}

1. Piotin M, Gailloud P, Bidaut L, et al. CT angiography, MR angiography and rotational digital subtraction angiography for volumetric assessment of intracranial aneurysms. An experimental study. Neuroradiology 2003;45:404-09

2. Bidaut LM, Laurent C, Piotin M, et al. Second-generation three-dimensional reconstruction for rotational three-dimensional angiography. Acad Radiol 1998;5:836-49

3. Ernemann UU, Gronewaller E, Duffner FB, et al. Influence of geometric and hemodynamic parameters on aneurysm visualization during three-dimensional rotational angiography: an in vitro study. AJNR Am J Neuroradiol 2003;24:597-603

4. Yamura M, Hirai T, Korogi Y, et al. Quantitative evaluation of measurement accuracy for three-dimensional angiography system using various phantoms. Radiat Med 2005;23:175-81

5. Heran NS, Song JK, Namba K, et al. The utility of DynaCT in neuroendovascular procedures. AJNR Am J Neuroradiol 2006;27:330-32

6. Sluzewski M, Rooij WJ, Slob MJ, et al. The relationship between aneurysm volume, packing, and compaction in 145 coiled cerebral aneurysms. Radiology 2004;231:653-58

7. Ford MD, Alperin N, Lee SH, et al. Characterization of volumetric flow rate waveforms in the normal internal carotid and vertebral arteries. Physiol Meas 2005;26:477-88

8. Wang Z-J, Hoffmann KR, Wang Z, et al. Contrast settling in cerebral aneurysm angiography. Phys Med Biol 2005;50:3171-81 